\title{
Ermächtigungsgesetzgebung in Deutschland zwischen Monarchie und Republik
}

\section{Enabling Acts in Germany from the Monarchy to the Republic}

This essay deals with the legal function of enabling acts, such as the enabling act of 1933, which has often been perceived as the central source of legality for the NS regime. However, the history of this specific kind of legislation began earlier, during World War I. From 1914 to 1923, approximately ten of these acts can be identified. Within this period, these acts were not the only means by which the German legislator dealt with states of emergency. However, enabling acts were the only way which permitted the legislators to tackle the problems related to the state of emergency without being bound to the constitutional framework of both the Constitution of the Reich (1871) and the Weimar Republic (1919) respectively. These acts mark fundamental challenges to a constitutional order and mostly transitional periods between political systems: at the end of the monarchy and of the republic.

Keywords: enabling acts - State of emergency - Weimar Constitution.

Unser Thema ist in Deutschland untrennbar mit dem Ermächtigungsgesetz vom 24. März 1933 verknüpft, welches die nationalsozialistische Herrschaft legitimieren sollte. Doch ist dies heute nur am Rande unser Thema. Das grundsätzliche Charakteristikum solcher Ermächtigungen ist die Abweichung der Rechtssetzungskompetenzen vom Text der geltenden Verfassung; es entsteht Verfassungsrecht außerhalb der Verfassungsurkunde. Genau darin liegt das Besondere der Ermächtigung. Für sie bedarf es stets einer Setzung von Verfassungsrecht. Ist eine Notkompetenz in der Verfassungsurkunde vorgesehen, bedarf es für sie oder ihre Ausübung keiner besonderen Ermächtigung durch Verfassungsrechtssetzung mehr. Sie ist deshalb hier auch nicht unser Thema. Ermächtigungsgesetzgebung fand sich in Deutschland nur kurze Zeit hindurch. Sie war eine staatsrechtliche Folge nicht des Konstitutionalismus, sondern seiner Krise und Auflösung im Weltkrieg. In der Republik wirkte sie ambivalent - tendenziell ver- fassungserhaltend in der Staatskrise des Jahres 1923, verfassungsvernichtend im Jahr 1933.

\section{Ouvertüre: Verfassungsdurchbrechung vor der Verfassung}

Am Anfang März 1919 amtierte die Weimarer Nationalversammlung gerade drei Wochen lang. Sie hatte kurz zuvor das Gesetz über die Vorläufige Reichsgewalt verabschiedet, ${ }^{1}$ welches die Konstituante zugleich als Gesetzgeber einsetzte $(\S 4)$ und die noch vom alten Reichstag verabschiedete Parlamentarisierung der Reichsregierung bestätigte ( 88 Abs. 2). Die Rechtssetzung sollte fortan ausschließlich auf seiner Grundlage erfolgen: Keine Gesetzgebung ohne

\footnotetext{
${ }^{1}$ ReichsG von 10. 2. 1919, RGBl. S. 169.
} 
Parlament! Jedenfalls insoweit war die alte Verfassung formell außer Kraft. ${ }^{2}$

In dieser staatsrechtlichen Übergangszeit rief der Präsident der Nationalversammlung einen Gesetzentwurf der Koalitionsparteien zur Durchführung der Waffenstillstandsbedingungen auf. ${ }^{3}$ Er betraf einen damals äußerst sensiblen Punkt: Es war das Deutsche Reich gewesen, das um den Waffenstillstand nachgesucht hatte; und dieser sowie die dafür maßgeblichen Bedingungen war immer nur kurzfristig von den Siegermächten formuliert worden. Die Materie war also sowohl im Fluss wie auch aus deutscher Sicht schwer vorhersehbar und daher schwer regelbar. Daher sollte die Reichsregierung ermächtigt werden, diejenigen wirtschaftlichen und finanziellen Maßnahmen mit gesetzlicher Wirkung anzuordnen, welche sich zur Durchführung der Waffenstillstandsbedingungen als notwendig erwiesen. Insoweit sollte die Gesetzgebung auf die Regierung übertragen werden. Darin lag eine zentrale Abweichung von der soeben beschlossenen vorläufigen Ordnung der Reichsgewalt, welche auf dem Gedanken der parlamentarischen Rechtssetzung basierte, ohne dieses förmlich zu ändern. Dessen zentrale Errungenschaft wurde also schon wenige Wochen nach ihren Erlass „durchbrochen“.

Die Materie war also verfassungsrechtlich von hoher Wichtigkeit. Dem kontrastierte ihre parlamentarische Behandlung. Der Präsident rief alle drei Lesungen nacheinander auf, doch meldete sich niemand zur Wort, und zwar weder von den einbringenden Koalitionsparteien noch von Seite der Opposition. Auch die bei Gesetzen übliche Überweisung an einen Ausschuss wurde - im Unterschied zur sonstigen Praxis der Nationalversammlung - nicht beantragt. Und nach dieser parlamentarischen Nichtbehandlung wurde das Gesetz einstimmig verabschiedet.

\footnotetext{
${ }^{2}$ Ihre definitive Aufhebung erfolgte durch Art. 178 WRV.

${ }^{3}$ Verhandlungen des Reichstags, Bd. 326, S. 416.
}

Das Haus ging zur weiteren Tagesordnung über. Das Procedere mag aus der Rückschau überraschen. Aus der Sicht von gut einem Drittel der Mitglieder der Versammlung konnte es jedoch auch als Déjà-vu-Erlebnis beschrieben werden. Sie waren nämlich schon dabei gewesen, als im Reichstag der Monarchie eine vergleichbare Szene stattgefunden hatte.

\section{Verfassungsdurchbrechung im Kaiserreich}

a) Knapp 5 Jahre zuvor, am 4. August 1914, war Ähnliches geschehen. Am Tag nach Ausbruch des Weltkriegs und im einsetzenden Augusterlebnis hatte der Reichstag dem Gesetz über die Ermächtigung des Bundesrats zu wirtschaftlichen Maßnahmen und über die Verlängerung der Fristen des Wechsel- und Scheckrechts im Falle kriegerischer Ereignisse zugestimmt. ${ }^{4}$ Das Gesetz ging über seinen unscheinbaren Titel weit hinaus und ermächtigte in $\S 3$ den Bundesrat, „während der Zeit des Krieges gesetzliche Maßnahmen anzuordnen, welche sich zur Abhilfe wirtschaftlicher Schädigungen als notwendig erweisen sollten". Auch hier fand sich ein Ermächtigungsgesetz, welches die Gesetzgebung in weitem Umfang auf nicht-parlamentarische Exekutiven delegierte. Und auch dies war ohne jede parlamentarische Beratung geschehen. In der damals in Deutschland weithin angenommenen Stunde höchster Not kannte offenbar auch der Reichstag keine Parteien mehr. Bekannter wurde ein anderer Beschluss vom gleichen Tag: Nahezu einstimmig billigte

\footnotetext{
${ }^{4}$ Gesetz über die Ermächtigung des Bundesrats zu wirtschaftlichen Maßnahmen und über die Verlängerung der Fristen des Wechsel- und Scheckrechts im Falle kriegerischer Ereignisse v. 4. 8. 1914, RGB1. S. 327.
} 
die Volksvertretung auch die notwendigen Kriegskredite. ${ }^{5}$

Augenscheinlich war den ohne Aussprache zustimmenden Abgeordneten die über den kriegsbedingten Augenblick weit hinausreichende staatsrechtliche Bedeutung ihres Schritts nicht bewusst gewesen. Denn die Ermächtigung war geeignet, das konstitutionelle Verfassungssystem aus den Angeln zu heben. Dessen rechtliche Neuerung hatte neben der Verfassungsbindung aller Staatsgewalt gerade darin bestanden, dass den Volksvertretungen ein Mitwirkungsrecht an der Gesetzgebung zuerkannt worden war. Mitwirkung an der Rechtssetzung war damals die einzige Form der Beteiligung der Volksvertretung an der Staatsgewalt. Und nun gab der Reichstag auch noch sein zentrales Mitwirkungsrecht „während der Dauer des Krieges" auf. Auffällig ist, dass dies ohne Kompensation geschah. Damit war die zentrale Errungenschaft der konstitutionellen Verfassungen in Deutschland für die Dauer des Krieges außer Kraft. Wie weit dieser Kompetenzverzicht reichte, zeigte am deutlichsten die Bestimmung zum AußerKraft-Treten: Dieses sollte durch kaiserliche Verordnung mit Zustimmung des Bundesrates geschehen. Die Adressaten der außerordentlichen Ermächtigung entschieden so über die zeitliche Dauer ihrer eigenen Ermächtigung.

Im Weltkrieg stand das Ermächtigungsgesetz als kriegsbedingtes Ausnahmerecht in Deutschland nicht allein. Neben der verfassungsdurchbrechenden Ermächtigung bestand der in der Verfassung von 1871 vorgesehene Belagerungszustand mit einem Notverordnungsrecht. Im Krieg standen so ein verfassungsdurchbrechendes Ermächtigungsgesetz und ein „Kriegszustandsrecht" nach der Verfassung nebeneinander.

Der Ausnahmezustand war so in Deutschland nicht bloß die viel zitierte „Stunde der Exekuti-

${ }^{5}$ Zum Ganzen: Verhandlungen des Reichstags, Bd. 306, S. $6 \mathrm{ff}$. $v^{\prime \prime}$. Er war zuerst einmal eine Stunde der weitreichenden Abdankung des Parlaments. Viel spricht dafür: Die tiefgreifende Verfassungskrise im Reich stand nicht am Ende des Krieges, sondern schon an dessen Anfang. Bereits der Kriegsausbruch und seine rechtliche Behandlung im August waren die Verfassungskrise. ${ }^{6}$

In der Folgezeit ergingen insgesamt 825 Bundesratsverordnungen zwischen dem 6. August 1914 und dem 7. November 1918.7 Sie betrafen ganz unterschiedliche Rechtsgebiete: Das Bewirtschaftungsrecht einschließlich des dafür notwendigen Organisationsrechts auf dem Weg zum bisweilen sog. „Kriegssozialismus“; das Währungs-, Finanz- und Steuerrecht, aber ohne das Recht der Kriegsanleihen und Steuererhöhungen; das allgemeine Zivilrecht, dabei ging es nicht nur um unmittelbar kriegsbedingte Frage wie Verschollenheit und Feindvermögen, sondern auch um die allgegenwärtige Wohnungsnot und das Mietrecht sowie Patente, Gebrauchs- und Geschmacksmuster; das Arbeitsund Sozialrecht, nicht bloß zum Arbeitsrecht der eingezogenen Soldaten, sondern auch die Lohnfestsetzung und Lohnpfändung, ferner die zukunftsträchtige Einführung eines allgemeinen staatlichen Arbeitsnachweises als Vorläufer der späteren Arbeitsverwaltung; schließlich das Gerichtsverfahrensrecht, wo es nicht bloß um kriegsbedingte Fragen von Fristen und Fristversäumnissen ging, sondern auch um die Einführung des schriftlichen Strafbefehls (ohne mündliche Verhandlung) und den Zahlungsbefehl

\footnotetext{
${ }^{6}$ Dies war den damals Beteiligten möglicherweise bewusst. Zu ihrer Haltung bei der informellen Gesetzesvorberatung FREHSE, Ermächtigungsgesetzgebung 12 ff.

${ }^{7}$ Darstellung bei HuBER, Deutsche Verfassungsgeschichte V, 69ff.; zur Entstehung des Gesetzes schon ebd. 62ff. Er hält die Ermächtigung für „in effektiver Weise sachlich begrenzt" (68) und sieht auch unter dem Ermächtigungsgesetz ein "gewisses Machtgleichgewicht zwischen dem anordnungsbefugten Bundesrat und dem kontrollbefugten Reichstag“ (69).
} 
(ohne vorherige gerichtliche Prüfung des Anspruchs). Beide Institute bestehen unter zum Teil anderen Namen noch in der Gegenwart. Folgt man der auch insoweit sehr exekutivfreundlichen Darstellung Ernst Rudolf Hubers, soll es nur in fünf Fällen Streitigkeiten über den Bezug von Verordnungen des Bundesrates zur Abwehr wirtschaftlicher Schädigungen gegeben haben. Dies waren sämtlich solche auf dem Gebiet des Prozessrechts.

Daneben war die reguläre Gesetzgebung aber nicht einfach beendet. Im Krieg stieg die Normproduktion mit dem Normbedarf. Und nach wie vor ergingen auch Gesetze. Doch waren sie selten (das wichtige Hilfsdienstgesetz zählte dazu) und im einstelligen Prozentbereich im Vergleich zur exekutiven Rechtssetzung. Rein quantitativ war die Rechtssetzung auf den Bundesrat übergegangen.

b) Der Weltkrieg wird gemeinhin als Verfassungskrise beschrieben, welche den Weg von der konstitutionellen zur parlamentarischen Monarchie eröffneten sollte. Dabei ist zunächst festzuhalten: Den späteren Parlamentarisierungstendenzen ging zunächst eine weitgehende Entparlamentarisierung des Reichs voraus. Neben der zivilen Reichsregierung zählte in der ersten Kriegshälfte der Reichstag zu den Verlierern der neuen Machtverhältnisse auf dem Weg zum ersten totalen Krieg.

Damit war die Frage nach der Rückkehr zur staatsrechtlichen Normalität aber nicht beantwortet, sondern eigentlich erst eröffnet. Sie war nicht mehr allein eine Frage des Rechts, sondern auch der Machtverhältnisse. Da Kaiser und Bundesrat auf dem Rückweg zur staatsrechtlichen Normalität eine Veto-Position besaßen ihre außerordentlichen Rechtssetzungsbefugnisse würden erst enden, wenn sie selbst zustimmten -, war die Situation dem Machtkampf um die Parlamentarisierung der Reichsregierung durchaus vergleichbar. Vielleicht zeigt sich hier die Bedeutung der Verfassungsdurchbrechung als Verfassungskrise am deutlichsten: Sie war nicht bloß geeignet, die Frage nach der Machtbalance nach der Reichsverfassung 1871 zu stellen, sondern zugleich die Verfassungsfrage selbst. Sie stellte sich als Frage nach den politischen Bedingungen einer möglichen Zustimmung der ermächtigten Organe zu ihrer eigenen Teilentmachtung - und damit zugleich als Frage nach der Reformfähigkeit der Monarchie und ihrer Verfassung. ${ }^{8}$ Hier war die Situation im Krieg keineswegs eindeutig: Bekanntlich entwickelten Reichswehrführung, konservative Gegner der zivilen Reichsregierungen und die Parteien im Reichstag ganz unterschiedliche, einander entgegenlaufende Verfassungspläne. Dem lag eine zumindest implizite Aufkündigung der Verfassungsloyalität nicht nur einzelner Parteien, sondern auch staats- und regierungstragender Eliten zugrunde. Die Anerkennung der geltenden Staatsform wurde ein bedingte, abhängig von äußeren Faktoren, und in deren Folge kündbar. Hier war der Weg des Reiches keineswegs vorgezeichnet, sondern sollte wesentlich vom Ausgang des Krieges und den sich daraus ergebenden Interessen- und Machtkonstellationen abhängen. Da wegen der einander gegenüberstehenden staatsrechtlichen Vetopositionen das politische System zur Selbstblockade neigte, war ein Ausweg aus der Krise im Kontext der geltenden Verfassung kaum noch denkbar. Dem Legitimationsverlust folgte der Geltungsverlust der Verfassung - und weil sich keine Seite für eine Rückkehr zur alten Ordnung stark machen wollte, fand sich gegen die Revolution im November 1918 auch keine Hand zu ihrer Verteidigung. Gewiss: Das Ermächtigungsgesetz war nicht die alleinige Ursache dieser Entwicklung, doch hatte es den Grundstein gelegt. Die Verfassungskrise war nicht das Kriegsende, sondern der Kriegsanfang gewesen - das sollte sich aber erst an dessen Ende besonders sinnfällig zeigen. Das Kriegsende brachte nicht allein das Ende

\footnotetext{
${ }^{8}$ Hierzu (wenn auch mit anderen Schwerpunkten) SCHÖNBERGER, Überholte Parlamentarisierung 623.
} 
der Ermächtigung, sondern das Ende der Verfassung, welche sie durchbrochen hatte.

\section{Verfassungsrecht vergeht, Ermächtigungsgesetzgebung besteht?}

Eine staatsrechtliche Neuerung schuf im Februar $1919 \S 10$ des Gesetzes über die vorläufige Reichsgewalt. Es setzte die Nationalversammlung als Gesetzgeber ein und ordnete an, dass neue Rechtsvorschriften nur noch nach den Regelungen dieses Gesetzes ergehen sollten. Jedenfalls insoweit galten die Bestimmungen der Reichsverfassung 1871 nicht mehr. Und die Ministerien gingen nunmehr davon aus, dass mit ihnen auch das ErmächtigungsG von 1914 nicht mehr gelten sollte - und zwar unabhängig von der Frage nach der Fortdauer oder dem Ende des Krieges. Offenbar bestand aber zugleich ein praktisches Bedürfnis nach exekutiver Normsetzung parallel zu der gesetzgebenden Nationalversammlung, die selbst ein großes und aus der Retrospektive erstaunliches Gesetzgebungsprogramm bewältigte. Noch mehr hätte sie nicht schaffen können. Da umgekehrt die Verordnunggebung eine tradierte Praxis war, die sich aus der Sicht der weiterhin amtierenden Ministerialbürokratien bewährt hatte, lag es nahe, an dem alten Instrumentarium festzuhalten. Dafür fehlte jedoch nunmehr die Rechtsgrundlage, so dass die Idee aufkam, eine neue Ermächtigung zu schaffen. ${ }^{9}$ Viel spricht dafür, dass dabei eher praktische Bedürfnisse als verfassungsrechtliche

\footnotetext{
${ }^{9}$ Woher die Idee kam, ist nicht klar auszumachen. In der Reichsregierung ist das Thema nicht behandelt worden. Der anhaltende Notstand war dort nahezu permanent präsent. Notverordnungsfragen wurden aber allein im Hinblick auf den zu schaffenden Art. 48 WRV diskutiert; s. SCHULZE, Akten der Reichskanzlei 467. $\mathrm{Zu}$ den Kabinettsangelegenheiten ebd. 1ff.; zu den Gegenständen der Beratungen Überblick ebd. XXXVIIIff.
}

Erwägungen im Mittelpunkt gestanden haben. Auf diese Weise wurden der Nationalversammlung drei Entwürfe zur vereinfachten Gesetzgebung vorgelegt, die innerhalb der ersten Monate ihrer Legislaturperiode erlassen wurden, darunter das bereits erwähnte Gesetz zur Durchführung der Waffenstillstandsbedingungen, welches in seinem Anwendungsbereich demjenigen des Gesetzes von 1914 in Teilen entsprach. ${ }^{10}$

Ein Blick in die drei Ermächtigungsgesetze der Konstituante zeigt einige Gemeinsamkeiten, aber auch zentrale Unterschiede. Alle drei Gesetze wurden von der Nationalversammlung verabschiedet, und zwar nach Erlass des Gesetzes über die Vorläufige Reichsgewalt, aber vor Inkrafttreten der WRV. Die Gesetzesbeschlüsse ergingen jeweils - wie 1914 - ohne vorherige Aussprache jedenfalls zur Ermächtigung, allenfalls wurde ihr Anlass debattiert. Die Einigung fand also - ganz wie 1914 - außerhalb des Plenums statt. ${ }^{11}$ Alle drei Gesetze ermächtigten die neue Reichsregierung zum Erlass von Maßnahmen mit gesetzlicher Wirkung, delegierten also einen Teil der Gesetzgebung auf die neue Regierung. Diese Ermächtigung war jeweils sachlich nur schwach begrenzt. Auch waren die Verordnungen der Nationalversammlung zur Kenntnis zu geben und auf deren Verlangen aufzuheben. Dies alles erinnerte stark an das Ermächtigungsgesetz von 1914.

Doch zeigten sich auch Unterschiede: Da war zunächst der Adressat der Ermächtigung, die Reichsregierung: Sie war als vorläufige Reichsgewalt parlamentarisiert, also der Nationalversammlung verantwortlich (§ 8 Abs. 2). Darin lag ein fundamentaler Unterschied gegenüber der Reichsverfassung 1871, welche die Regierung als kaiserliche mit nur bescheidenen parlamentarischen Kontrollmöglichkeiten verfasst hatte. Nunmehr sollte die Rechtssetzung von einer

\footnotetext{
${ }^{10}$ ReichsG v. 6. 3. 1919, RGB1. S. 286; dazu Verhandlungen des Reichstages, Bd. 326, S. 416.

${ }^{11}$ FREHSE, Ermächtigungsgesetzgebung 12ff., $51 \mathrm{ff}$.
} 
parlamentarischen auf eine parlamentarisch verantwortliche Instanz übertragen werden. Darin lag ein rechtlich bedeutsamer Unterschied. Ein weiterer findet sich in der zeitlichen Begrenzung: Die Übertragung sollte allein für die Dauer der Nationalversammlung gelten und danach enden, sie war also durch die Legislaturperiode der Weimarer Konstituante begrenzt. Das Ende der Ermächtigung war also nun klar begrenzt, ihr Auslaufen an keine weiteren amtlichen Feststellungen mehr geknüpft. Im April 1919 fanden sich im 3. Ermächtigungsgesetz staatsrechtlich bedeutsame Neuerungen: Dazu zählte die Einsetzung eines Ausschusses, ohne dessen Zustimmung die Notgesetze nicht in Kraft treten konnten. Darin lag eine ansatzweise Parlamentarisierung der Notrechtskompetenzen: Der Erlass notwendiger Maßnahmen war nun nicht mehr allein Regierungsangelegenheit, sondern sollte schon im Verfahren und nicht erst nach ihrem Inkrafttreten Gegenstand parlamentarischer Kenntnis und Beratung werden.

Zusammenfassend bleibt festzuhalten: Die RV 1871 trat außer Kraft, die Ermächtigungsgesetzgebung galt zwar nicht fort, lebte aber wieder auf. So eindeutig hier Kontinuität erkennbar war, so fallen auch staatsrechtlich erhebliche Neuerungen auf. Sehr deutlich zeigten dies die sich rasch herausbildenden Unterschiede zwischen den Ermächtigungsgesetzen vom März und April. Dieser Wandel innerhalb spricht stark dafür, die Ermächtigungsgesetzgebung als typische staatsrechtliche Übergangserscheinung zu deuten.

Die Rechtssetzungspraxis bestätigte das Bedürfnis nach außerordentlicher Rechtssetzung. Von den neuen Ermächtigungen wurde vielfältiger, im Einzelnen unterschiedlicher Gebrauch gemacht. ${ }^{12}$ Einige der besonders umstrittenen Notmaßnahmen sollten weit über die Legislaturperiode der Konstituante hinaus fortgelten.

${ }^{12}$ Im einzelnen ebd. 50f., 55, 64ff.
Das galt namentlich für die Verordnungen über den Vorläufigen Reichswirtschaftsrat (4. Mai 1923), die Errichtung des Reichsamts für Arbeitsvermittlung (5. Mai 1920), und über das Reichswirtschaftsgericht (21. Mai 1920). Die maßgeblichen Aushandlungsprozesse fanden wegen der breiten Mehrheit der Koalitionsparteien im Reichstag konsensual statt. Ein Aufhebungsverlagen des Plenums fand sich in keinem Fall.

Die Kontinuitätsthese nach dem Motto „Verfassungsrecht vergeht - Ermächtigungsgesetzgebung besteht" ist in dieser Form nicht aufrechtzuerhalten. Sie konnte allenfalls für das damals empfundene Bedürfnis nach außerordentlicher Rechtssetzung angenommen werden. Aber die daraus hergeleiteten Rechtsfolgen befanden sich in raschem Wandel.

\section{Ermächtigungs- gesetzgebung durch Verfassungsdurchbrechung}

Nach Inkrafttreten der WRV, Auflösung der Nationalversammlung und dem Zusammentritt des ersten Reichstags war u.a. die auflösende Bedingung der bis dahin ergangenen Ermächtigungsgesetze eingetreten: Sie verloren automatisch ihre Wirksamkeit. Fortan konnten auf ihrer Grundlage keine gesetzesvertretenden Verordnungen mehr ergehen. Die zuvor erlassenen gesetzesvertretenden Regelungen blieben jedoch von dem Ereignis unberührt und bis zu ihrer Aufhebung in Kraft. Doch hatten sich zu diesem Zeitpunkt die faktischen und rechtlichen Rahmenbedingungen in mehrerer Hinsicht verändert. ${ }^{13}$

\footnotetext{
13 Älterer Überblick bei HUBER, Deutsche Verfassungsgeschichte $437 \mathrm{ff}$.
} 


\section{Der verfassungsrechtliche Rahmen}

Das Inkrafttreten der Weimarer Verfassung hat das politische System der Republik zwar auf eine stabile Rechtsgrundlage gestellt, doch blieben die politischen Voraussetzungen seiner Existenz weiterhin schwankend. Wenn es dafür irgend eines Beleges bedurfte, so war dieser schon in den Umständen der Auflösung der Nationalversammlung und der Ansetzung der Reichstagswahl zu sehen: Der Kapp-Putsch hatte gezeigt, dass nicht nur ein erheblicher Teil der Bürger, sondern auch der bewaffneten Macht immerhin nunmehr der Armee der Republik nicht auf dem Boden der neuen Verfassung standen und sogar bereit waren, gegen diese mit der Waffe zu kämpfen. Tatsächlich hatte der Abschluss der Verfassunggebung nicht befriedend gewirkt. Hinzu kamen die wirtschaftlichen Notstände, welche fortdauern sollten: Reparationen, Kriegskredite, Kriegsfolgelasten und Konversionskosten. Sie waren eine zentrale Ursache für die Hyperinflation des Jahres 1923. Dieses Jahr stellte die Existenzfrage der Republik.

Das Inkrafttreten der Weimarer Reichsverfassung im August 1919 hatte die ordentliche wie auch die außerordentliche Rechtssetzung auf eine neue Rechtsgrundlage gestellt. Die WRV kannte allein das reguläre Verfahren der Gesetzgebung durch den Reichstag. Einzige Form des Staatsnotrechts waren die Sonderrechte des Reichspräsidenten nicht der Regierung! - nach Art. 48 WRV, welche auf eine erhebliche Störung oder Gefährdung der öffentlichen Sicherheit und Ordnung abstellten. Sollte aus den damals aktuellen Krisen außerordentlicher rechtlicher Regelungsbedarf entstehen, so konnte dieser nach der Intention der Nationalversammlung, dem Text des Art. 48 WRV und der damals vorherrschenden Auslegung des „Diktaturartikels“ nur teilweise und unter intensiver Rechtsfortbildung mit dessen Instrumentarium bewältigt werden. So weit war man damals noch nicht.

Daraus konnte immerhin die Idee entstehen: Wenn der faktische Notstand fortdauerte, dar- aus ein außerordentlicher Rechtssetzungsbedarf entstand, die alten außerordentlichen Rechtssetzungsrechte ihre Gültigkeit verloren und die neue Verfassung keine entsprechenden Notkompetenzen mehr enthielt, so lag es nahe, $L \ddot{o}-$ sungen außerhalb der Verfassung bzw. an ihr vorbei zu suchen. Da eine entsprechende Regelung in der WRV nicht vorhanden war, konnte eine Lösung nur durch Verfassungsänderung oder durch Verfassungsdurchbrechung erfolgen. Auf jeden Fall setzte dies einen weiteren Akt der Verfassungsrechtssetzung voraus.

Da es zu einer Änderung des Textes der WRV bis 1933 insoweit nicht kommen sollte, kam dazu nur der Weg des Ermächtigungsgesetzes in Betracht, also die Verfassungsdurchbrechung. Wenn ein Gesetz von den verfassungsändernden Organen im Verfassungsänderungsverfahren mit den für Verfassungsänderungen notwendigen Mehrheiten beschlossen wurde, so konnte darin eine Änderung des Verfassungsrechts ohne Änderung des Verfassungstexts gesehen werden. Die Lehre von der Differenzierung der Normebenen und dem Vorrang der Verfassung damals wurde in Deutschland - anders als in Österreich - auch von den verfassungsloyalen Autoren kaum vertreten. ${ }^{14}$

\section{Ermächtigungsgesetzgebung in der Republik}

a) Nach Inkrafttreten der WRV ergingen in den Jahren 1920-1923 insgesamt fünf Ermächtigungsgesetze:

\footnotetext{
${ }^{14}$ Eher oberflächliche Bestandsaufnahme bei SCHAU, Verhältnis von Verfassung und einfachem Recht. Zur damaligen Position Kelsens Kelsen, Allgemeine Staatslehre 248ff. Er betont zwar die Verfassungsbindung des Gesetzgebers, nimmt dort aber zum Problem der verfassungsdurchbrechenden Gesetze nicht explizit Stellung.
} 
Die beiden Gesetze von 1920 und $1921^{15}$ knüpften tatbestandlich unmittelbar an das letzte Ermächtigungsgesetz der Nationalversammlung an. Zur Begründung wurde auch darauf hingewiesen, dass das Bedürfnis nach außerordentlicher Rechtssetzung auch nach dem Auseinandergehen der Nationalversammlung noch fortbestehe. Das Gesetz war auch ansonsten mit der Vorgängerregelung identisch: Der mitwirkungsberechtigte parlamentarische Ausschuss wurde mit denselben Kompetenzen erneut eingerichtet.

Das Reichsnotgesetz von $1923^{16}$ erging nach längerer ermächtigungsloser Zeit als Reaktion auf die französische Besetzung des Ruhrgebiets wegen Reparationsstreitigkeiten. Das Gesetzeswerk stand ganz im Zeichen der herzustellenden „Einheitsfront" im "Abwehrkampf". Die heute nur noch schwer erkennbare politische und emotionale Bedeutung dieser Auseinandersetzung erinnerte in Einzelheiten an das Augusterlebnis; und erst der Ausgang des Ruhrkampfes bewirkte die innere Einsicht der Deutschen in Kriegsniederlage und die Unausweichlichkeit von Versailles. ${ }^{17}$ Die Ermächtigung bezog sich auf unterschiedliche wirtschaftliche und soziale Auswirkungen "fremder Einwirkung" auf Deutschland und umfassende wirtschaftspolitische Regelungen zur Regelung des Wirtschafts- und Zahlungsverkehrs. Der Reichstag war nicht mitwirkend eingebunden und auf das nachträgliche Aufhebungsverlangen beschränkt. Das Gesetz verlor mit der Einstellung

\footnotetext{
${ }^{15}$ Gesetze v. 3. 8. 1920, RGBl. S. 1493 und vom 6. 2. 1921, RGBl. S. 139, dazu Verhandlungen des Reichstages, Bd. 346, S. 1854ff.; Bd. 347, S. 2186ff.; RGBl. S. 147, dazu Verhandlungen des Reichstages, Bd. 358, S. 9947ff.; RGB1. S. 1920, 1483, dazu Verhandlungen des Reichstages, Bd. 344, S. 250, 461; HUBER, Deutsche Verfassungsgeschichte VII, 160f.; FREHSE, Ermächtigungsgesetzgebung $67 \mathrm{ff}$.

${ }^{16}$ RGBl. S. 147, dazu Verhandlungen des Reichstages Bd. 358, S. $9947 f f$.

${ }^{17}$ Aus jüngerer Zeit GEYER, Zwischen Krieg und Nachkrieg.
}

des Ruhrkampfs Ende September seine Anwendbarkeit. Wenn es in der Republik eine existenzielle Verfassungskrise gab, dann hier. Das Ermächtigungsgesetz wirkte als ein als notwendig empfundener Krisenbewältigungsmechanismus. Einmal mehr bestätigte sich: Ermächtigungsgesetze setzten Verfassungskrisen voraus.

Das Erste Reichsermächtigungsgesetz von $1923^{18}$ zur Bekämpfung der Folgen der Hyperinflation. Waren hier schon die materiellen Grenzen der Ermächtigung kaum erkennbar, so galt dies erst recht für die formellen Vorkehrungen: Erstmals wurde weder die Zustimmung eines Reichstagsausschusses noch diejenige des Reichsrats vorgesehen. Es blieb bei dem nachträglichen parlamentarischen Aufhebungsverlangen. Die Mitwirkung der Abgeordnete war aber dadurch gesichert, dass der parteipolitische Bestand der Koalition als (Fort-)Geltungsbedingung normiert wurde. Das parlamentarische Vertrauen wurde so unterstellt und brauchte nicht in jedem Einzelfall gesucht zu werden. Und da die Koalition schon drei Wochen später zerbrach, endete auch die Ermächtigung alsbald. Die überaus kurze Dauer der Ermächtigung ist von den Ministerien genutzt worden, um Schubladenentwürfe umzusetzen. Eine Folge waren parlamentarische Auseinandersetzungen über Notwendigkeit und Zulässigkeit einzelner Verordnungen. Sie setzten jedoch erst ein, als das Gesetz schon außer Kraft war.

Das Zweite Reichsermächtigungsgesetz von $1923^{19}$ folgte in der Ausgestaltung der Vorgängerregelung. Noch mehr als zuvor ergingen Schubladenverordnungen wie die sog. Emmingersche Justizreform, die Fürsorgepflichtverordnung (die bis 1961 als Vorläuferin des BundessozialhilfeG fortgelten sollte) und die Schaffung der

\footnotetext{
${ }^{18}$ RGBl. S. 943; dazu Verhandlungen des Reichstages, Bd. 361, S. $12033 \mathrm{ff}$.

${ }^{19}$ Gesetz v. 8. 12. 1923, RGB1. S. 1179; dazu Verhandlungen des Reichstages, Bd.361, S. 12296ff., 12352ff., $12359 f f$.
} 
Deutschen Reichsbahn. Sie wurden - neben anderen gesetzlichen und administrativen Maßnahmen - eine Grundlage für den erstaunlichen Stabilisierungserfolg, der im 1. Halbjahr 1924 eintreten sollte. Dessenungeachtet fanden Bemühungen um eine Verlängerung der zuletzt genannten Ermächtigungen keine ausreichende parlamentarische Unterstützung. Damit fand das Instrument sein Ende. ${ }^{20}$

b) Angesichts der Größe und Vielfältigkeit der Krise der Republik im Jahr 1923 ist erstaunlich, wie relativ rasch und wirkungsvoll sie Ende 1923/Anfang 1924 beigelegt werden konnte. Die Bewältigung der schweren Staatskrise brachte zugleich das Ende der Ermächtigungsgesetzgebung in der Republik. Schon 1921 waren Zweifel an ihrer Notwendigkeit aufgekommen. Inzwischen griffen immer mehr "Diktaturverordnungen" nach Art. 48 WRV auch auf wirtschaftliche Fragen über. Zur „Öffentlichen Sicherheit“ wurde fortan auch die Funktionsfähigkeit der Finanz-, Wirtschafts- und Sozialordnung gerechnet. ${ }^{21}$ Die sich rasch durchsetzende neue Auffassung ließ erkennen: Wenn und weil es in der Weimarer Verfassung nach der neuen Auslegung anwendbare Instrumente, Verfahren und Rechtsformen gab, um der Notsituation Herr zu werden, schwand der Bedarf nach außerordentlichen Ermächtigungen an der Verfassung vorbei. Was mit der Verfassung geleistet werden konnte, brauchte keine Verfassungsdurchbrechung. Und selbst diese war immer weniger nötig: In der mittleren Phase der Republik blieb die Zahl der Diktaturverordnungen einstellig, und darunter befand sich eine Reihe von Ver-

\footnotetext{
${ }^{20}$ Die beiden weiteren damals noch sog. Ermächtigungsgesetze über die vorläufige Anwendung völkerrechtlicher von Wirtschaftsabkommen vor ihrer Ratifikation (ReichsG v. 10.7. 1926, RGBl. II 421; v. 14. 7. 1927, RGBl. II 466) betrafen Einzelfälle möglicher Kollision völker- und staatsrechtlicher Rechtsfragen ohne grundsätzliche Verschiebung der Gewalten.

${ }^{21}$ Näher dazu GusY, Weimar 55f.
}

ordnungen, welche allein der Aufhebung älterer Verordnungen galten.

Zusammenfassend fanden sich für das Ende der Ermächtigungsgesetzgebung seit 1924 also mehrere Gründe:

Die rasche Beruhigung und Stabilisierung der Verhältnisse in der mittleren Phase der Republik beendeten deren Gründungskrisen und damit das Bedürfnis nach außerordentlicher Rechtssetzung.

Die in der mittleren Phase vielfach amtierenden Minderheitskabinette konnten für außerordentliche Rechtssetzung nicht mehr die erforderliche ZweiDrittel-Mehrheit mobilisieren. Der Bedarf war zu gering, ihre Unterstützung zu schmal.

Zudem erschien Bedarf nach Ermächtigungen kaum erkennbar, seit aufgrund der erweiternden Auslegung der Reichspräsident und Art. 48 Abs. 2 WRV als hinreichend erschienen, im Rahmen der Normalverfassung und nicht erst infolge weiterer Akte der Verfassungsrechtssetzung entstehende Krisen zu meistern. Dies sollte denn auch in der Weltwirtschaftskrise ab 1930 geschehen.

Im Zuge der Politik Hindenburgs, die bald nach seinem Amtsantritt auf die Herausbildung eines Präsidialregimes gerichtet war, erschien zudem eine Ermächtigung der parlamentarischen Reichsregierung weder notwendig noch erwünscht. ${ }^{22}$

Keine Rolle spielte bei dem Ende der Ermächtigungsgesetzgebung demgegenüber die angedeutete Kritik der Staatsrechtswissenschaft an den Verfassungsdurchbrechungen. Sie wurde erst nach 1924 hinreichend vernehmbar. Und die verfassungsdurchbrechende Gesetzgebung endete zwar hinsichtlich der Ermächtigungsgesetze, nicht aber insgesamt: Auf anderen Gebieten

${ }^{22}$ Grundlegend MOMMSEN, Verspielte Freiheit 414ff., der den ganzen Abschnitt unter den bezeichnenden Titel „Um das Überleben des parlamentarischen Systems" stellt. 
sollten sich auch in Zukunft weitere Durchbrechungen finden. ${ }^{23}$

\section{Coda: Das Ermächtigungs- gesetz von 24. März 1933}

Das Gesetz vom 8. Dezember 1923 sollte das letzte Ermächtigungsgesetz der Republik sein. Aber es war nicht das letzte Ermächtigungsgesetz in Deutschland überhaupt. Bekanntlich hat der Reichstag am 24. März 1933 das „Gesetz zur Behebung der Not von Volk und Reich" verabschiedet. $^{24}$ Im Gesetzgebungsverfahren wurde auch auf die Tradition der Ermächtigungsgesetze in der Republik hingewiesen. Dabei unterschied sich das Gesetz in nahezu allen Fragen von seinen republikanischen Vorgängern. Es enthielt keine materielle Begrenzung der Ermächtigung, sondern umfasste alle denkbaren Reichsgesetze ohne Rücksicht auf Notwendigkeit oder Dringlichkeit einschließlich verfassungsändernder Regelungen. Beides kannte in der Republik kein Vorbild. Eine parlamentarische Mitwirkung oder Kontrolle war - erstmals seit der Übergangszeit 1920 - nicht vorgesehen, selbst das nachträgliche Aufhebungsverlangen war entfallen. Es war auf vier Jahre befristet wesentlich länger als alle anderen Ermächtigungen in Friedenszeiten - und allein durch den Fortbestand der gegenwärtigen Reichsregierung auflösend bedingt. Selbst in der Existenzkrise der Republik 1923 waren derart weitreichende Ermächtigungen mit derart marginalen Kontrollen für einen derart langen Zeitraum nicht erteilt worden. Auch wenn immerhin geregelt war, dass auch im Verfahren der WRV noch Gesetze zulässig sein sollten: Dies war die Abdankung des soeben gewählten Reichstags für eine ganze Legislaturperiode.

\footnotetext{
${ }^{23}$ Überblick bei LOEWENSTEIN, Erscheinungsformen $75 f$.

${ }^{24}$ RGBl. S. 141.
}

Auch ex post ist das letzte Ermächtigungsgesetz unter Rückgriff auf seine republikanischen Vorgänger immer wieder gerechtfertigt, seine Legalität behauptet worden. ${ }^{25}$ Dies ermöglichte den Apologeten zugleich den Aufweis angeblicher "Mängel“ der WRV, welche die legale Machtübertragung an die Nationalsozialisten zugelassen habe. Die Legalitätsthese behauptete die Vereinbarkeit des Ermächtigungsgesetzes mit der WRV. Doch hatten die Legalitätsbehauptungen der NSDAP schon in der Republik ausschließlich taktisch-propagandistische Funktion eingenommen. ${ }^{26}$ Und die Praxis des Jahres 1933 widersprach schon dem zeitgenössischen staatsrechtlichen Erkenntnisstand. Das gilt etwa für die Behauptung, das erforderliche Zustimmungsquorum sei erreicht worden, selbst wenn man die 81 Mandate der verhafteten bzw. verfolgten und daher abwesenden KPDAbgeordneten zu den 94 opponierenden Stimmen der SPD hinzurechne. Die Vereinbarkeit des Gesetzes mit der WRV ließe sich damit aber allenfalls dann begründen, wenn die Immunitätsregel des Art. 37 WRV die betroffenen Abgeordneten ausschließlich in ihrem individuellen Interesse, nicht aber mit ihnen zugleich die Funktionsfähigkeit und Abstimmungsfreiheit des Reichstags insgesamt garantierte. Doch war letztere Auffassung in der Republik faktisch

\footnotetext{
${ }^{25}$ So mit ausführlichen Nachweisen FREHSE, Ermöchtigungsgesetzgebung 145ff., 161ff., 168ff. Die Diskussionen um die Vereinbarkeit des Ermächtigungsgesetzes vom 24.3. 1933 mit der WRV war eine typische ex-post-Diskussion: Sie konnte erst nach 1945 aufkommen und blieb daher ausschließlich eine historische. Aus der älteren Literatur ARNDT, Zur Rechtsgültigkeit 240; THOMA, Urteilsanmerkung 142f; BRECHT, Vorspiel zum Schweigen 133ff. Später SCHNEIDER, Ermächtigungsgesetz; DERS., Reichsverfassung 177, Rz. 85ff.; s.a. GRAWERT, Nationalsozialistische Herrschaft 238, Rz. 5; Heuss, Machtergreifung; MATTHÉE, Legalität des Ermächtigungsgesetzes; Weimarer Reichsverfassung 151f, 400 ff.; zur leerlaufenden WRV seit 1930 ebd., $403 \mathrm{ff}$.

${ }^{26}$ RÜFFLER, Vom Münchener Landfriedensbruch.
} 
konkurrenzlos. ${ }^{27}$ Die von der NSDAP ganz wesentlich mitverursachten bürgerkriegsähnlichen Zustände des Jahres 1932 und der "Wahlkampf“ des Jahres 1933 hatten mit Legalität jedenfalls im Sinne der WRV nichts zu tun. Und die einschüchternden Begleitumstände der Reichstagssitzung vom 23. März 1933 hatten es auch nicht. ${ }^{28}$ Das Ermächtigungsgesetz war nicht "legal“ im Sinne der Weimarer Verfassung. Und im Reichsrat wurden die Stimmen einzelner Länder von dazu nicht berechtigten Reichskommissaren abgegeben. Dass die fortan amtierenden NS-Regierungen sich alsbald auch nicht mehr an das Ermächtigungsgesetz hielten, fügte dem Ganzen nur noch ein charakteristisches Apercu hinzu.

Dies alles geschah unter Missbrauch der Verfassung ${ }^{29}$ des Normal- bzw. des Ausnahmezustandes. ${ }^{30}$ Die nationalsozialistische Herrschaft war nicht Ausdruck demokratischer Legalität im Rahmen der WRV, sondern vielmehr Folge ihres tiefsten Niedergangs. Die Legalitätsthese stößt inzwischen zu Recht weithin auf Ablehnung. ${ }^{31}$ Sie diente ganz überwiegend der Verantwortungsverlagerung für die nationalsozialistische Herrschaft auf Faktoren, die sich nach 1945 nicht wehren konn-

\footnotetext{
${ }^{27}$ ANSCHÜTZ, Verfassung des Deutschen Reichs 232: nicht Privileg des Abgeordneten, sondern Privileg des Parlaments. DoHNA, Insbesondere: Redefreiheit 445f.: "nicht um ein Vorrecht des einzelnen Abgeordneten, sondern um ein Schutzrecht des Hauses“ im „Interesse der Legislative am ungestörten Fortgang der parlamentarischen Arbeit“. Daher könne ein Verzicht des einzelnen Abgeordneten auf jenen Schutz die Genehmigung des Hauses nicht ersetzen.

${ }^{28}$ Historisch MORSEY, Ermächtigungsgesetz 129ff.; HEuss, Machtergreifung.

${ }^{29}$ So die zusammenfassende These von BüTTNER, Überforderte Republik 498ff.

${ }^{30}$ Einzelne Aspekte hierzu bei DREIER, Macht und Ohnmacht.

${ }^{31}$ Ablehnend etwa STOLLEIS, Geschichte des öffentlichen Rechts 246f., 317ff.; DREIER, Die drei gängigsten Irrtümer 1154ff.; GusY, Weimarer Reichsverfassung 151f., $459 \mathrm{ff}$.
}

ten. Zu ihnen zählte an prominentester Stelle die WRV.

Es bleibt also dabei: Das Ermächtigungsgesetz vom Dezember 1923 war das letzte Gesetz dieser Art der Republik. Dasjenige von 1933 setzte Leerlauf bzw. Ende der Verfassung voraus und war zugleich ein Gründungsdokument des NSStaates.

\section{Ein verfassungshistorisches und verfassungsrechtliches Fazit}

Der Grundgedanke des Verfassungsstaates erst recht in seiner parlamentarisch-demokratischen Variante - liegt darin, die Einhaltung der Verfassung durch die Staatsorgane nicht von deren subjektivem Willen zur Verfassung, sondern von kompetenz-, organisations- und verfahrensrechtlichen Normen abhängig zu machen. Dieser Grundgedanke wird jedenfalls geschwächt, wenn die entsprechenden Regelungen in Stunden der Not einfach durchbrochen werden können. In diesem Sinne wirken sie wie ein Stück Selbstpreisgabe des Verfassungsgedankens und des Verfassungsrechts. Einerseits waren sie Teil der Notmaßnahmen in der groBen Staatskrise 1923 und damit Teil des Instrumentariums, mit welchen die Republik gerettet wurde. Andererseits ist die Einsicht, dass die Ermächtigungsgesetze in der Republik den Glauben an die Wirksamkeit des Parlamentarismus (weiter) beeinträchtigt haben, ein Grundakkord der historischen Literatur. ${ }^{32}$ Mit den Instrumenten, mit welchen die Republik im Jahr 1923/24 gerettet worden ist, ist sie im Jahr 1933 zerstört worden. Ermächtigungsgesetze wirkten also einmal (1914) verfassungsgefährdend, einmal (1923, neben anderen Maßnahmen) verfassungserhaltend, einmal verfassungsvernichtend (1933).

${ }^{32}$ FREHSE, Ermächtigungsgesetzgebung 135. 
Die verfassungsrechtliche Würdigung der Ermächtigungsgesetzgebung in Deutschland ist eindeutig negativ. Sie steht vollständig unter dem Eindruck des Ermächtigungsgesetzes von 1933. Es gilt als verfassungszerstörend und ist im Grundgesetz ausdrücklich untersagt. Das gilt sowohl formell gegenüber der Verfassungsdurchbrechung (Art. 79 Abs. 1 GG) wie auch materiell durch die Unantastbarkeit der Grundsätze der gewaltenteilenden Demokratie (Art. 79 Abs. 3 GG). Dazu wird auch die gesetzesvertretende Rechtssetzung unter Ausschluss des Parlaments gerechnet. ${ }^{33}$ Außerordentliche Rechtsetzung ist nur durch und allein unter genau konstitutionalisierten Voraussetzungen in einem gleichfalls geregelten vereinfachten Verfahren zulässig (Art. 81, 115d GG).

\section{Korrespondenz:}

Prof. Dr. Christoph GUSY

Universität Bielefeld

Fakultät für Rechtswissenschaft

Postfach 100131

33501 Bielefeld

Deutschland

christoph.gusy@uni-bielefeld.de

\footnotetext{
33 S. etwa DreIER, in: Ders., Art. 79 Rz. 15: „keine legale Revolution“. Drastisch EvERS, in: KAHL, WALDHOFF, WALTER, Art. 79 Abs. 3 Rz. 112: „Illegalisierung der Verfassungsvernichtung durch gesetzesförmliche Verfassungsänderung." Noch drastischer im Parlamentarischen Rat Abg. Dehler, „Barriere [...] in dem Willen, einer Revolution die Maske der Legalität zu nehmen“, zit.n. DoEmMING, FÜsSLeIN, MATZ, Entstehungsgeschichte 586:
}

\section{Abkürzungen:}

Siehe das allgemeine Abkürzungsverzeichnis: [http://www.rechtsgeschichte.at/files/abk.pdf]

\section{Literatur:}

Gerhard ANSchüTZ, Die Verfassung des Deutschen Reichs vom 11. August 1919 (Aalen 141933).

Gerhard ANschüTZ, Richard ThOMA (Hgg.), Handbuch des Deutschen Staatsrechts, Bd. I (Heidelberg 1930).

Adolf ARNDT, Zur Rechtsgültigkeit nationalsozialistischer Gesetz, in: Deutsche Rechts-Zeitschrift 3 (1948) $240 f$.

Arnold BRECHT, Vorspiel zum Schweigen. Das Ende der deutschen Republik (Wien 1948).

Ursula BÜTTNER, Die überforderte und missbrauchte Republik (Stuttgart 2008).

Klaus-Berto DoEMmING, Werner Rudolf FüsSLEIN, Werner MATZ, Entstehungsgeschichte der Artikel des Grundgesetzes, im Auftrage der Abwicklungsstelle des Parlamentarischen Rates und des Bundesministers des Innern auf Grund der Verhandlungen des Parlamentarischen Rates (= Jahrbuch des öffentlichen Rechts der Gegenwart. NF 1, Tübingen 1951).

Alexander Graf zu DOHNA, Insbesondere: Redefreiheit, Immunität und Zeugnisverweigerungsrecht, in: Gerhard ANSCHÜTZ, Richard ThOMA (Hgg.), Handbuch des Deutschen Staatsrechts, Bd. I (Tübingen 1930) $445 f$.

Horst DREIER, Die drei gängigsten Irrtümer über die Weimarer Reichsverfassung, in: Merkur 63 (2009) $1151 \mathrm{ff}$.

Ders. (Hg.), Macht und Ohnmacht des Grundgesetzes. Sechs Würzburger Vorträge zu 60 Jahren Verfassung (Berlin 2009).

DERS., in: DERS. (Hg.), Grundgesetz-Kommentar, Bd. II: Art. 20-82 (Tübingen ${ }^{32015) .}$

Hans-Ulrich Evers, in: Wolfgang KAHL, Christian WaldhofF, Christian Walter (Hgg.), Bonner Kommentar zum Grundgesetz, 192. AL, August 2018 (Heidelberg 1950ff.).

Michael FREHSE, Ermächtigungsgesetzgebung im Deutschen Reich 1914-1933 (Pfaffenweiler 1985).

Michael GeYER, Zwischen Krieg und Nachkrieg. Die deutsche Revolution 1918/19 im Zeichen blockierter Transnationalität, in: Alexander GALLUS (Hg.), Die vergessene Revolution von 18/1919 (Göttingen 2005) 187-222. 
Rolf GRAWERT, Die nationalsozialistische Herrschaft, in: Josef IsENSEE, Paul KIRCHHOF (Hgg.), Handbuch des Staatsrechts der Bundesrepublik Deutschland, Bd. 1: Historische Grundlagen (Heidelberg ${ }^{32003)}$ 235-286.

Christoph Gusy, Weimar. Die wehrlose Republik? (Tübingen 1991).

Christoph GusY, Die Weimarer Reichsverfassung (Tübingen 1997).

Theodor Heuss, Die Machtergreifung und das Ermächtigungsgesetz Zwei nachgelassene Kapitel der Erinnerungen, hg. v. Eberhard PIKART (Tübingen 1967).

Ernst-Rudolf HUBER, Deutsche Verfassungsgeschichte seit 1789, Bd. V-VI (Stuttgart 1980, 1981).

Hans KelSEN, Allgemeine Staatslehre (Wien 1925).

Karl LOEWENSTEIN, Erscheinungsformen der Verfassungsänderung (Aalen 1931).

Ulrich MATTHÉE, Die Legalität des Ermächtigungsgesetzes vom 24. 3. 1933 und die Schranken der Verfassungsrevision in der WRV (Kiel 1971).

Hans Mommsen, Die verspielte Freiheit. Der Weg der Republik von Weimar in den Untergang 1918 bis 1933 (Berlin 1990).

Rudolf MORSEY, (Hg.) Das Ermächtigungsgesetz vom 24. März 1933 (Göttingen 21992).
Klaus RÜFfLER, Vom Münchener Landfriedensbruch bis zum Mord von Potempa (Frankfurt am Main u.a. 1994).

Götz-Friedrich SCHAU, Das Verhältnis von Verfassung und einfachem Recht in der Staatsrechtslehre der Weimarer Republik (Frankfurt am Main 2002).

Hans SCHNEIDER, Das Ermächtigungsgesetz vom 24. 3. 1933, in: Vierteljahreshefte für Zeitgeschichte 1 (1955) 197-221..

Hans SCHNEIDER, Die Reichsverfassung vom 11. August 1919, in: Josef IsENSEE, Paul KirCHHOF (Hgg.), Handbuch des Staatsrechts der Bundesrepublik Deutschland, Bd. I: Historische Grundlagen (Heidelberg ${ }^{32003)}$ 177-234.

Christoph SCHÖNBERGER, Die überholte Parlamentarisierung. Einflußgewinnung und fehlende Herrschaftsfähgkeit des Reichstags im sich demokratisierenden Kaiserreich, in: HZ 272 (2001) 623-666.

Hagen SCHUlzE (Bearb.), Akten der Reichskanzlei. Das Kabinett Scheidemann (Boppard am Rhein 1971).

Manfred STOLLEIS, Geschichte des öffentlichen Rechts in Deutschland, Bd. 3: Staats- und Verwaltungsrechtswissenschaft in Republik und Diktatur 1914 bis 1945 (München 1999).

Richard THOMA, Urteilsanmerkung zu OLG Tübingen, in: DRZ 3 (1948) 142f. 